\title{
Resilience as a Protective Factor for Suicidal Ideation among Korean Workers
}

\author{
Sun Mi Kim¹, Hye Ri Kim¹, Kyoung Joon Min', Seo-Koo Yoo², \\ Young-Chul Shin ${ }^{3,4}$, Eun-Jin Kim ${ }^{3,4}$, and Sang Won Jeon ${ }^{3,4 凶}$ \\ 'Department of Psychiatry, College of Medicine, Chung-Ang University, Seoul, Republic of Korea \\ ${ }^{2}$ School of Social Welfare, Soongsil University, Seoul, Republic of Korea \\ ${ }^{3}$ Department of Psychiatry, Kangbuk Samsung Hospital, Sungkyunkwan University School of Medicine, Seoul, Republic of Korea \\ ${ }^{4}$ Workplace Mental Health Institute, Kangbuk Samsung Hospital, Seoul, Republic of Korea
}

Objective This study aimed to investigate resilience as a protective factor for suicidality among Korean workers.

Methods Participants were workers from 26 organizations in Korea, aged 18 to 63 years, who completed a self-reported questionnaire comprising items on sociodemographic factors, job stress, resilience, and suicidality. Completed questionnaires were collected from 4,405 persons, with 4,389 valid responses. Hierarchical logistic regression analyses were performed using suicidality as the dependent variable.

Results Results of the logistic regression analyses indicated that among the sociodemographic factors, older age, being female, and being single were statistically significantly associated with suicidal ideation. A high level of job stress, depressive mood, and anxiety, in addition to a short job duration, were also associated with suicidal ideation. Resilience was a significant protective factor for suicidal ideation after adjusting for all other variables. Older age and high anxiety levels were associated with having a suicide plan among participants with suicidal ideation. The association of resilience with suicide plans and suicide attempts was non-significant.

Conclusion In this study, we found that a high level of resilience was associated with a low incidence of suicidal ideation in Korean workers. Based on these results, we suggest the need to develop and implement interventions to improve resilience in the workplace, thereby protecting workers, especially those with a high level of work stress, from suicidal ideation.

Psychiatry Investig 2020;17(2):147-156

Key Words Psychological resilience, Workplaces, Occupational health, Protective factor, Suicidal ideation.

\section{INTRODUCTION}

Suicide is a leading cause of death in working-age populations. In Korea, suicide was the highest cause of death among people in their 20s and 30s, and the second highest cause of death among those in their 40s and 50s in 2017. ${ }^{\circ}$ Of the Korean workers who died in 2017, the deaths of $13.69 \%$ were due to suicide. ${ }^{1}$ It is known that those who complete suicide have generally previously made 10 to 20 attempts. ${ }^{2}$ Suicide and re-

Received: March 26, 2019 Revised: August 1, 2019

Accepted: November 27, 2019

$\triangle$ Correspondence: Sang Won Jeon, MD, PhD

Department of Psychiatry, Kangbuk Samsung Hospital, Sungkyunkwan University School of Medicine, 29 Saemunan-ro, Jongno-gu, Seoul 03181, Republic of Korea

Tel: +82-2-2001-2073, Fax: +82-2-2001-2211

E-mail: sangwonyda@hanmail.net

(a) This is an Open Access article distributed under the terms of the Creative Commons Attribution Non-Commercial License (https://creativecommons.org/licenses/bync/4.0) which permits unrestricted non-commercial use, distribution, and reproduction in any medium, provided the original work is properly cited. lated behaviors can have huge costs with regard to workers' productivity and welfare. Not only completed suicides but also non-fatal suicidal ideation, plans, and attempts can cause serious economic burdens in the workplace. ${ }^{2}$ Therefore, it is important to identify risk and protective factors for suicidal behaviors at work and use them to prevent suicide.

Workers' suicide is a result of complex interactions between individual vulnerabilities (such as mental health problems), stressful working conditions, and living conditions (including familial and social stressors). Previous studies have associated increased suicidality with high work stress; ${ }^{3}$ adverse working conditions such as a monotonous job, high responsibility, and high job strain; ${ }^{4}$ and workplace bullying. ${ }^{5}$ In a recent systematic review and meta-analysis, exposure to poor job support from supervisors and coworkers and work insecurity were significantly related to suicidal ideation. ${ }^{6}$ In addition, lack of job control and low job support from supervisors and coworkers were significantly related to suicide completion. ${ }^{6}$ Although 
previous studies have been limited by their cross-sectional nature, there is growing evidence to suggest an association between poor employment-related psychosocial conditions and suicidality. Psychosocial stressors in the workplace can affect mental health by interacting with vulnerability and protective factors. However, there have been few studies on the relationship between individual protective factors and occupational stress and suicidality in the domain of job-related mental health.

Resilience refers to an individual's ability to successfully cope with stressors and changes in the environment and maintain psychological well-being despite adversity. The concept of resilience first received attention with observations of people who recovered and adapted better than others after experiencing severe stressful events. ${ }^{7}$ At first, resilience was regarded as a fixed personality trait. ${ }^{8}$ However, in animal and human studies, it has been observed that coping ability is strengthened in response to environmental risks and protection. ${ }^{8}$ Therefore, recently, resilience has come to be regarded not as a "fixed trait" but a "dynamic process" that changes and improves with environmental stimuli. ${ }^{8}$ As the concept of resilience has developed, studies have been conducted to investigate its relationship with mental illness in people exposed to specific stressors and to prevent mental illness by providing training or fostering an environment that can enhance resilience.

In resilience research, the workplace has attracted attention as an environment where individual resilience can be developed and promoted as a protective factor for mental health problems. In previous studies, resilience was reported to be a protective factor for depression and posttraumatic stress disorder in certain occupational groups known to be at high risk of trauma or those who experience occupational stress. ${ }^{9-11}$ A systematic review of 14 studies from 2003 to 2014 reported that training in improving resilience in the workplace could improve mental health problems such as stress, depression, and anxiety. ${ }^{12}$ However, few studies have investigated the association between resilience and suicidality among workers. In the general population, there has been increasing interest in the association between resilience and suicidality, with studies associating a low resilience score with an increased risk of suicide attempts. ${ }^{13}$ In a lifespan analysis study, a low level of resilience was shown to predict greater suicidality in all ages. ${ }^{14}$ A review of studies on moderators affecting suicidality proposed a "buffering hypothesis" that can explain how resilience reduces suicide risk. ${ }^{15}$ As per the buffering hypothesis, resilience can moderate the impact of the existing risk of suicidality, such as a negative attributional style, excessive perfectionism, and hopelessness. ${ }^{15}$

Therefore, in this study, we aimed to investigate the association between resilience and suicidality among workers. Further, we aimed to determine the influence of resilience on different levels of suicidality, which is a comprehensive term including suicidal ideation (thoughts of ending one's life), suicide plans (strategies for how to end one's life), and suicide attempts (usually non-lethal self-harm behavior, while some are willing to die). In the study population, we hypothesized that 1) high resilience is related to a low incidence of suicidal ideation and 2) high resilience is related to a low incidence of suicide plans among participants with suicidal ideation and a low incidence of suicide attempts among participants with suicide plans.

\section{METHODS}

\section{Study participants}

This study was part of the Kangbuk Samsung Workplace Mental Health Study, a cross-sectional study of male and female Koreans aged 18 to 63 years who attended a mental health checkup program at the Workplace Mental Health Institute, Kangbuk Samsung Hospital in Seoul, Korea. Participants comprised workers from 26 companies and local government organizations, and voluntarily participated in mental health examinations upon invitation from their companies: 10 companies in the fields of service (three providing educational services, two providing health services, three providing business services, and two hotels), two companies in the field of transportation and communications (one transportation company and one broadcasting company), three companies in the field of finance, insurance, and real estate (three insurance companies), three companies in the field of construction, three companies in the field of manufacturing, three companies in the field of wholesale trade, and two public administrations, based on the United States' Standard Industrial Classification division structure. ${ }^{16}$

The present study was conducted between June 2015 and April 2017. From the initial 4,405 respondents, we excluded those with incomplete questionnaires or missing sociodemographic information. The final sample size was 4,389 $(2,432$ males, 1,957 females). The study was approved by the Institutional Review Board of Kangbuk Samsung Hospital (IRB no. KBSMC 2013-01-217), which waived the informed consent requirement as we only used de-identified data routinely collected in the workplace mental health screening checkups.

\section{Measures}

\section{Suicidality}

Suicidality was assessed using a self-reported dichotomous question, the response options for which were "Yes" or "No." The question is part of the Korea National Health and Nutrition Examination Survey conducted annually by the Korean government to investigate the level of public health and compile statistics to establish and evaluate public health policy. ${ }^{17}$ 
Participants' responses were categorized into groups according to their suicide status during the previous year as follows: 1) those who had never considered suicide, 2) those who had experienced suicidal ideation, 3 ) those who had made suicide plans, and 4) those who had attempted suicide. To assess suicidal ideation, participants were asked whether they had ever seriously wanted to commit suicide during the past year ("Over the last year, have you ever felt you would be better off dead?"), with response options of "Yes" or "No." To determine the existence of a suicide plan, participants were asked whether they had ever made a specific plan for suicide during the past year ("Over the last year, have you ever planned how to commit suicide?"), with response options of "Yes" or "No." The presence of a suicide attempt was also measured by a question regarding if they had ever attempted self-harm or suicide during the previous year ("Over the last year, have you ever attempted selfharm or suicide?"), with response options of "Yes" or "No."

\section{Sociodemographic factors}

The sociodemographic factors collected for the purpose of this study were age, gender, educational level, marital status, job grade, and years at current job. Educational level was categorized into two groups, with those with master's and doctorate degrees referred to as the highly educated group. Marital status was delineated as married or single (being single included never married, separated, divorced, and widowed individuals), and job grade as low (members of staff or assistant managers) or high (executive team members, general managers, deputy general managers, or managers).

\section{Occupational stress}

Occupational stress was measured using the short form of the Korean Occupational Stress Scale (KOSS-SF) developed to measure employees' job stress in Korean workplaces. ${ }^{18}$ The KOSS-SF is a self-reported questionnaire comprising 24 items measured on a four-point Likert scale (1 to 4). In this study, Cronbach's alpha for the KOSS-SF was 0.643 . Some papers have asserted that an acceptable threshold for Cronbach's alpha is typically $\geq 0.70 .{ }^{19}$ Previously, the KOSS-SF has demonstrated good internal consistency and validity within seven subscales: job demand, lack of job control, interpersonal conflict, job insecurity, organizational system, insufficient reward, and organizational climate. ${ }^{18}$ However, in this study, the analyses that follow use the overall KOSS-SF score, not those of the seven subscales, to determine internal consistency; therefore, Cronbach's alpha for the KOSS-SF is relatively low.

\section{Psychiatric symptoms}

Depression and anxiety levels were assessed using the 20item Center for Epidemiological Studies Depression (CES-D) scale $^{20,21}$ and the 21-item Beck Anxiety Inventory (BAI), ${ }^{22}$ respectively. Both are self-reported questionnaires with responses measured on a four-point Likert scale ranging from 0 to 3 points, and both have been confirmed to have high internal consistency and validity. ${ }^{20-22}$ In this study, Cronbach's alphas of the CES-D and BAI were 0.861 and 0.937 , respectively, indicating high levels of internal consistency in the context of our sample.

\section{Resilience}

The Korean version of the Connor-Davidson Resilience Scale (K-CD-RISC) was used to measure resilience. ${ }^{23}$ The self-reported K-CD-RISC consists of 25 items measured on a five-point Likert scale ranging from 0 to 4 points. Previously, the K-CDRISC demonstrated good reliability and validity (Cronbach's alpha $=0.93$, test-retest reliability $=0.93) .{ }^{23}$ In this study, Cronbach's alpha of the K-CD-RISC was 0.995 , indicating a high level of internal consistency in the context of our sample.

\section{Statistical analysis}

The distribution of sociodemographic and psychological characteristics across the study population was calculated. For univariate analyses, we used Mann-Whitney-U tests to compare means of continuous variables because of non-normal distribution, and chi-square tests to compare proportions of categorical variables between participants with and without suicidal ideation.

To determine the influence of resilience on the suicidality of workers, we performed hierarchical logistic regression analyses. First, a hierarchical logistic regression analysis of all participants was conducted with suicidal ideation as the dependent variable. Second, we conducted a hierarchical logistic regression analysis of participants with suicidal ideation using a suicide plan as the dependent variable. Third, we conducted a hierarchical logistic regression analysis of participants with a suicide plan using a suicide attempt as the dependent variable.

The model design was identical for all three analyses. In model 1, the association of sociodemographic factors and suicidality was tested. Occupational stress was added in model 2 to test the associations of occupational stress beyond the effects of sociodemographic factors. Psychiatric symptoms were added in model 3 to test the associations of depression and anxiety beyond the effects of sociodemographic factors and occupational stress. Finally, resilience was added in model 4 to test the associations of resilience beyond the effects of sociodemographic factors, occupational stress, and psychiatric symptoms. Statistical significance was set at $\alpha=0.05$ (two-sided). We used the Complex Samples module of the PASW statistics software package, version 19 (SPSS Inc., Chicago, IL, USA) to conduct all analyses. 


\section{RESULTS}

\section{Characteristics of the participants}

Sociodemographic factors, occupational stress, psychiatric symptoms, resilience, and suicidality status are described in
Table 1. The mean age of the participants was $39.6 \pm 10.2$ years, and $11.9 \%$ of them had a high educational level. The mean job duration was $13.0 \pm 10.4$ years, and $35.6 \%$ of the study population was in high-grade jobs. A total of $36.0 \%$ of the study population was single (never married, separated, divorced, or

Table 1. Sociodemographic and psychological characteristics of the study participants $(N=4,389)$

\begin{tabular}{|c|c|c|c|c|c|c|}
\hline \multirow{3}{*}{ Variables } & \multirow{3}{*}{ Min/Max } & \multirow{3}{*}{$\begin{array}{c}\text { Mean } \pm S D, N(\%) \\
\text { Total }\end{array}$} & \multicolumn{4}{|c|}{ Univariate analyses* } \\
\hline & & & \multicolumn{2}{|c|}{ Mean $\pm S D, N(\%)$} & \multicolumn{2}{|c|}{ Statistics } \\
\hline & & & No-SI group & SI group & $\mathrm{z} / \chi^{2}$ & $\mathrm{p}$ \\
\hline Age (years) & $18 / 63$ & $39.6 \pm 10.2$ & $40.3 \pm 10.3$ & $37.0 \pm 9.4$ & -8.641 & $<0.001$ \\
\hline Job duration (years) & $0 / 42$ & $13.0 \pm 10.4$ & $13.7 \pm 10.5$ & $10.5 \pm 9.5$ & -8.216 & $<0.001$ \\
\hline KOSS-SF & $1.2 / 92.9$ & $43.3 \pm 12.2$ & $41.7 \pm 11.7$ & $49.5 \pm 12.3$ & -16.435 & $<0.001$ \\
\hline CES-D & $0.0 / 58.0$ & $10.2 \pm 9.6$ & $8.3 \pm 8.1$ & $17.6 \pm 11.6$ & -23.805 & $<0.001$ \\
\hline BAI & $0.0 / 63.0$ & $7.2 \pm 8.2$ & $5.7 \pm 6.8$ & $13.4 \pm 10.4$ & -23.332 & $<0.001$ \\
\hline K-CD-RISC & $0.0 / 100.0$ & $63.6 \pm 16.0$ & $65.9 \pm 15.3$ & $54.6 \pm 15.8$ & -18.404 & $<0.001$ \\
\hline Gender (female) & & $1957(44.6)$ & $1449(41.3)$ & $508(57.7)$ & 76.127 & $<0.001$ \\
\hline \multicolumn{7}{|l|}{ Educational level } \\
\hline Below high school & & $516(11.8)$ & $399(11.4)$ & $117(13.3)$ & 26.197 & $<0.001$ \\
\hline College degree & & $1019(23.2)$ & $810(23.1)$ & $209(23.7)$ & & \\
\hline University degree & & $2330(53.1)$ & $1837(52.4)$ & $493(56.0)$ & & \\
\hline Master's degree & & $475(10.8)$ & 419 (11.9) & $56(6.4)$ & & \\
\hline Doctorate degree & & $49(1.1)$ & $43(1.2)$ & $6(0.7)$ & & \\
\hline Educational level (high) $^{\dagger}$ & & $524(11.9)$ & $462(13.2)$ & $62(7.0)$ & 25.188 & $<0.001$ \\
\hline \multicolumn{7}{|l|}{ Marital status } \\
\hline Never married & & $1482(33.8)$ & $1070(30.5)$ & $412(46.8)$ & 116.799 & $<0.001$ \\
\hline Married & & $2810(64.0)$ & $2379(67.8)$ & $431(48.9)$ & & \\
\hline Separated & & $13(0.3)$ & $8(0.2)$ & $5(0.6)$ & & \\
\hline Divorced & & $72(1.6)$ & $43(1.2)$ & $29(3.3)$ & & \\
\hline Widowed & & $12(0.3)$ & $8(0.2)$ & $4(0.5)$ & & \\
\hline Marital status (single) $\ddagger$ & & $1579(36.0)$ & $1129(32.2)$ & $450(51.1)$ & 109.143 & $<0.001$ \\
\hline \multicolumn{7}{|l|}{ Job grade } \\
\hline Executive team & & $165(3.8)$ & $143(4.1)$ & $22(2.5)$ & 99.005 & $<0.001$ \\
\hline General manager & & $436(9.9)$ & $402(11.5)$ & $34(3.9)$ & & \\
\hline Deputy general manager & & $403(9.2)$ & $346(9.9)$ & $57(6.5)$ & & \\
\hline Manager & & $557(12.7)$ & $474(13.5)$ & $83(9.4)$ & & \\
\hline Assistant manager & & $613(14.0)$ & $484(13.8)$ & $129(14.6)$ & & \\
\hline Staff & & $2215(50.4)$ & $1659(47.3)$ & $556(63.1)$ & & \\
\hline Job grade (high)§ & & $1561(35.6)$ & $1365(38.9)$ & $196(22.2)$ & 85.321 & $<0.001$ \\
\hline Suicidal ideation (yes) & - & $881(20.1)$ & & & & \\
\hline Suicide plan (yes) & - & $251(5.7)$ & & & & \\
\hline Suicide attempts (yes) & - & $66(1.5)$ & & & & \\
\hline
\end{tabular}

*univariate analyses (Mann-Whitney-U tests for continuous variables and chi-square tests for categorical variables) were conducted with suicidal ideation as the dependent variable, †educational level (high) includes master's and doctorate degrees, ${ }^{ \pm}$marital status (single) includes never married, separated, divorced, and widowed, §job grade (high) includes executive team members, general managers, deputy general managers, and managers. SD: standard deviation, No-SI group: participants without suicidal ideation, SI group: participants with suicidal ideation, KOSS-SF: Korean Occupational Stress Scale-Short Form, CES-D: Center for Epidemiologic Studies Depression scale, BAI: Beck Anxiety Inventory, K-CD-RISC: Korean version of the Connor-Davidson Resilience Scale 
widowed). The mean KOSS-SF score was $43.3 \pm 12.2$, and mean CES-D and BAI scores were $10.2 \pm 9.6$ and $7.2 \pm 8.2$, respectively. The mean K-CD-RISC score was $63.6 \pm 16.0$. In addition, $20.1 \%$ of the study participants $(n=881)$ reported having experienced suicidal ideation during the previous year, 5.7\% ( $\mathrm{n}=$ 251) reported having made a suicide plan during the previous year, and $1.5 \%(n=66)$ reported having attempted self-harm or suicide. In univariate analyses, compared to the participants without suicidal ideation, the participants with suicidal ideation were more likely to be younger $(\mathrm{z}=-8.641, \mathrm{p}<0.001)$, female $\left(\chi^{2}=76.127, \mathrm{p}<0.001\right)$, and single $\left(\chi^{2}=109.143, \mathrm{p}<0.001\right)$, and have a lower educational level $\left(\chi^{2}=25.188, \mathrm{p}<0.001\right)$, shorter job duration $(\mathrm{z}=-8.216, \mathrm{p}<0.001)$ and lower job grade $\left(\chi^{2}=\right.$ $85.321, \mathrm{p}<0.001)$. In addition, the participants with suicidal ideation were more likely to show higher KOSS-SF ( $z=-16.435$, $\mathrm{p}<0.001)$, CES-D $(\mathrm{z}=-23.805, \mathrm{p}<0.001)$, and BAI $(\mathrm{z}=-23.332$, $\mathrm{p}<0.001)$ scores and lower K-CD-RISC scores $(\mathrm{z}=-18.404, \mathrm{p}<$ 0.001 ) compared to those without suicidal ideation.

\section{Results of hierarchical logistic regression analyses of all participants with suicidal ideation as the dependent variable}

The results of the hierarchical logistic regression analysis that tested the association of suicidal ideation with sociodemographic factors, occupational stress, psychiatric symptoms, and resilience are summarized in Table 2. The results of all four models of the hierarchical logistic regression revealed significant overall model fit. In the analysis of model 4, both model $\chi^{2}(786.586, \mathrm{p}<0.001)$ and the Nagelkerke $\mathrm{R}^{2}$ value $(0.259$, explaining about $25.9 \%$ of the variance in the dependent variable) indicated that the model was adequate to explain suicidal ideation. Examining the practical usefulness of the model according to classification accuracy, 10 variables in analysis model 4 considerably enhanced its explanation accuracy to $82.4 \%$ for the group membership of the dependent variable. Wald statistics were used to see if each indicator had a significant individual relationship with suicidal ideation. Among the independent variables, being female and being single were statistically associated with suicidal ideation. In addition, age, KOSS-SF score, CES-D score, and BAI score were statistically associated with suicidal ideation. Furthermore, job duration

Table 2. Results of hierarchical logistic regression analyses of all participants with suicidal ideation as the dependent variable $(\mathrm{N}=4,389)$

\begin{tabular}{|c|c|c|c|c|c|c|c|c|c|c|c|c|}
\hline \multirow{2}{*}{ Independent variables } & \multicolumn{3}{|c|}{ Model 1} & \multicolumn{3}{|c|}{ Model 2} & \multicolumn{3}{|c|}{ Model 3} & \multicolumn{3}{|c|}{ Model 4} \\
\hline & B & Wald & OR & $\mathrm{B}$ & Wald & OR & B & Wald & OR & B & Wald & OR \\
\hline \multicolumn{13}{|l|}{ Sociodemographic factors } \\
\hline Age (years) & 0.018 & 5.900 & $1.018^{*}$ & 0.027 & 12.739 & $1.028^{* * *}$ & 0.035 & 18.506 & $1.036^{* * *}$ & 0.037 & 20.175 & $1.038^{* * *}$ \\
\hline Gender (female) & 0.403 & 21.967 & $1.496^{* * *}$ & 0.335 & 14.187 & $1.399^{* * *}$ & 0.296 & 9.727 & $1.344^{* *}$ & 0.274 & 8.204 & $1.315^{* *}$ \\
\hline Marital status (single) & 0.531 & 28.971 & $1.700^{* * *}$ & 0.500 & 24.735 & $1.648^{* * *}$ & 0.394 & 13.521 & $1.482^{* * *}$ & 0.382 & 12.607 & $1.464^{* * *}$ \\
\hline Educational level (high) & -0.442 & 8.882 & $0.643^{* *}$ & -0.395 & 6.784 & $0.674^{* *}$ & -0.371 & 5.453 & $0.690^{*}$ & -0.278 & 3.006 & 0.758 \\
\hline Job duration & -0.019 & 8.854 & $0.981^{* *}$ & -0.027 & 15.602 & $0.974^{* * *}$ & -0.025 & 12.110 & $0.976^{* *}$ & -0.025 & 12.466 & $0.975^{* * *}$ \\
\hline Job grade (high) & -0.358 & 10.724 & $0.699^{* *}$ & -0.270 & 5.801 & $0.763^{*}$ & -0.263 & 4.991 & $0.769^{*}$ & -0.185 & 2.433 & 0.831 \\
\hline \multicolumn{13}{|l|}{ Job stress } \\
\hline KOSS-SF & & & & 0.051 & 215.992 & $1.052^{* * *}$ & 0.021 & 29.089 & $1.021^{* * *}$ & 0.015 & 12.601 & $1.015^{* * *}$ \\
\hline \multicolumn{13}{|l|}{ Psychiatric symptoms } \\
\hline CES-D & & & & & & & 0.050 & 81.426 & $1.051^{* * *}$ & 0.045 & 61.986 & $1.046^{* * *}$ \\
\hline BAI & & & & & & & 0.044 & 45.778 & $1.045^{* * *}$ & 0.042 & 41.046 & $1.042^{* * *}$ \\
\hline \multicolumn{13}{|l|}{ Resilience } \\
\hline K-CD-RISC & & & & & & & & & & -0.020 & 39.754 & $0.980^{* * *}$ \\
\hline \multicolumn{13}{|l|}{ Statistics of the model } \\
\hline$-2 \mathrm{LL}$ & \multicolumn{3}{|c|}{4224.935} & \multicolumn{3}{|c|}{3990.276} & \multicolumn{3}{|c|}{3654.221} & \multicolumn{3}{|c|}{3613.908} \\
\hline Model $\chi^{2}$ & \multicolumn{3}{|c|}{$175.559(\mathrm{df}=6)^{* * *}$} & \multicolumn{3}{|c|}{$410.218(\mathrm{df}=7)^{* * *}$} & \multicolumn{3}{|c|}{$746.273(\mathrm{df}=9)^{* * *}$} & \multicolumn{3}{|c|}{$786.586(\mathrm{df}=10)^{* * *}$} \\
\hline Step $\chi^{2}$ & \multicolumn{3}{|c|}{$175.559(\mathrm{df}=6)^{* * *}$} & \multicolumn{3}{|c|}{$234.658(\mathrm{df}=1)^{* * *}$} & \multicolumn{3}{|c|}{$336.056(\mathrm{df}=2)^{* * *}$} & \multicolumn{3}{|c|}{$40.313(\mathrm{df}=1)^{* * *}$} \\
\hline Nagelkerke $\mathrm{R}^{2}$ & \multicolumn{3}{|c|}{0.062} & \multicolumn{3}{|c|}{0.141} & \multicolumn{3}{|c|}{0.247} & \multicolumn{3}{|c|}{0.259} \\
\hline Classification accuracy & \multicolumn{3}{|c|}{79.9} & \multicolumn{3}{|c|}{80.2} & \multicolumn{3}{|c|}{81.7} & \multicolumn{3}{|c|}{82.4} \\
\hline
\end{tabular}


and K-CD-RISC score were statistically associated with suicidal ideation. High educational level and high job grade were statistically associated with suicidal ideation in analysis models 1, 2, and 3. However, educational level and job grade became statistically non-significant in analysis model 4 . After accounting for the influence of other variables, the odds ratio of 0.980 in the K-CD-RISC means that a positive increase in one unit of the K-CD-RISC decreased the probability of suicidal ideation by 0.98 times.

\section{Results of hierarchical logistic regression analyses of participants with suicidal ideation with a suicide plan as the dependent variable}

The results of the hierarchical logistic regression analysis that tested the association of having a suicide plan with sociodemographic factors, occupational stress, psychiatric symptoms, and resilience among participants with suicidal ideation are summarized in Table 3. The results of all four models of the hierarchical logistic regression revealed significant overall model fit. In analysis model 4, both model $\chi^{2}(87.408, \mathrm{p}<0.001)$ and the Nagelkerke $\mathrm{R}^{2}$ value $(0.137$, explaining about $13.7 \%$ of the variance in the dependent variable) indicated that the analysis model was adequate to explain having a suicide plan. Examining the practical usefulness of the model according to classification accuracy, 10 variables in analysis model 4 considerably enhanced its explanation accuracy to $74.5 \%$ for the group membership of the dependent variable. Wald statistics were used to determine if each indicator had a significant individual relationship with having a suicide plan. Among the independent variables, age and BAI score were statistically associated with having a suicide plan in analysis model 4 . The KOSSSF score was statistically associated with having a suicide plan in analysis models 2 and 3. In addition, job duration was statistically associated with having a suicide plan in analysis model 2. Furthermore, CES-D score was statistically associated with having a suicide plan in analysis model 3. However, KOSS-SF score, job duration, and CES-D score became statistically nonsignificant in analysis model 4 . In addition, the K-CD-RISC score did not demonstrate a statistically significant influence on having a suicide plan among participants with suicidal ideation.

Table 3. Results of hierarchical logistic regression analyses of participants with suicidal ideation with a suicide plan as the dependent variable $(\mathrm{N}=881)$

\begin{tabular}{|c|c|c|c|c|c|c|c|c|c|c|c|c|}
\hline \multirow{2}{*}{ Independent variables } & \multicolumn{3}{|c|}{ Model 1} & \multicolumn{3}{|c|}{ Model 2} & \multicolumn{3}{|c|}{ Model 3} & \multicolumn{3}{|c|}{ Model 4} \\
\hline & B & Wald & OR & B & Wald & OR & B & Wald & OR & B & Wald & OR \\
\hline \multicolumn{13}{|l|}{ Sociodemographic factors } \\
\hline Age (years) & 0.018 & 1.349 & 1.018 & 0.028 & 3.187 & 1.029 & 0.038 & 5.406 & $1.039^{*}$ & 0.039 & 5.571 & $1.040^{*}$ \\
\hline Gender (female) & -0.001 & 0.000 & 0.999 & 0.079 & 0.191 & 1.082 & 0.066 & 0.124 & 1.068 & 0.057 & 0.093 & 1.059 \\
\hline Marital status (single) & 0.330 & 3.093 & 1.391 & 0.277 & 2.144 & 1.319 & 0.233 & 1.405 & 1.262 & 0.229 & 1.367 & 1.258 \\
\hline Educational level (high) & -0.439 & 1.606 & 0.645 & -0.486 & 1.890 & 0.615 & -0.593 & 2.645 & 0.552 & -0.566 & 2.384 & 0.568 \\
\hline Job duration & -0.019 & 1.851 & 0.981 & -0.028 & 3.778 & $0.973^{*}$ & -0.028 & 3.579 & 0.973 & -0.028 & 3.659 & 0.972 \\
\hline Job grade (high) & 0.033 & 0.021 & 1.033 & 0.116 & 0.251 & 1.123 & 0.071 & 0.087 & 1.073 & 0.077 & 0.104 & 1.081 \\
\hline \multicolumn{13}{|l|}{ Job stress } \\
\hline KOSS-SF & & & & 0.032 & 25.587 & $1.033^{* * *}$ & 0.014 & 4.069 & $1.015^{*}$ & 0.013 & 3.190 & 1.013 \\
\hline \multicolumn{13}{|l|}{ Psychiatric symptoms } \\
\hline CES-D & & & & & & & 0.020 & 3.855 & $1.020^{*}$ & 0.018 & 3.047 & 1.018 \\
\hline BAI & & & & & & & 0.040 & 13.705 & $1.041^{* * *}$ & 0.040 & 13.679 & $1.041^{* * *}$ \\
\hline \multicolumn{13}{|l|}{ Resilience } \\
\hline K-CD-RISC & & & & & & & & & & -0.005 & 0.730 & 0.995 \\
\hline \multicolumn{13}{|l|}{ Statistics of the model } \\
\hline$-2 \mathrm{LL}$ & \multicolumn{3}{|c|}{1027.762} & \multicolumn{3}{|c|}{1001.330} & \multicolumn{3}{|c|}{949.143} & \multicolumn{3}{|c|}{948.411} \\
\hline Model $\chi^{2}$ & \multicolumn{3}{|c|}{$8.058(\mathrm{df}=6)$} & \multicolumn{3}{|c|}{$34.490(\mathrm{df}=7)^{* * *}$} & \multicolumn{3}{|c|}{$86.677(\mathrm{df}=9)^{* * *}$} & \multicolumn{3}{|c|}{$87.408(\mathrm{df}=10)^{* * *}$} \\
\hline Step $\chi^{2}$ & \multicolumn{3}{|c|}{$8.058(\mathrm{df}=6)$} & \multicolumn{3}{|c|}{$26.432(\mathrm{df}=1)^{* * *}$} & \multicolumn{3}{|c|}{$52.187(\mathrm{df}=2)^{* * *}$} & \multicolumn{3}{|c|}{$0.732(\mathrm{df}=1)$} \\
\hline Nagelkerke $\mathrm{R}^{2}$ & \multicolumn{3}{|c|}{0.013} & \multicolumn{3}{|c|}{0.056} & \multicolumn{3}{|c|}{0.136} & \multicolumn{3}{|c|}{0.137} \\
\hline Classification accuracy & \multicolumn{3}{|c|}{72.5} & \multicolumn{3}{|c|}{73.2} & \multicolumn{3}{|c|}{74.5} & \multicolumn{3}{|c|}{74.5} \\
\hline
\end{tabular}

${ }^{*} \mathrm{p}<0.05,{ }^{* *} \mathrm{p}<0.01,{ }^{* * *} \mathrm{p}<0.001$. OR: odd ratio, KOSS-SF: Korean Occupational Stress Scale-Short Form, CES-D: Center for Epidemiologic Studies Depression scale, BAI: Beck Anxiety Inventory, K-CD-RISC: Korean version of the Connor-Davidson Resilience Scale 


\section{Results of hierarchical logistic regression analysis of participants with a suicide plan with self-harm and suicide attempts as the dependent variable}

The results of the hierarchical logistic regression analysis that tested the association of suicide attempts with sociodemographic factors, occupational stress, psychiatric symptoms, and resilience among participants with a suicide plan are summarized in Table 4. The results of all four models in the hierarchical logistic regression revealed non-significant overall model fit. K-CD-RISC score did not demonstrate a statistically significant influence on suicide attempts among participants with a suicide plan.

\section{DISCUSSION}

To summarize the results, among the sociodemographic factors, older age, being female, and being single were statistically significantly associated with suicidal ideation. A high level of job stress, depressive mood, and anxiety, in addition to a short job duration, were also associated with suicidal ideation. Resilience was a significant protective factor for suicidal ideation after adjusting for all other variables. Older age and high anxiety levels were associated with having a suicide plan among participants with suicidal ideation. The association of resilience with suicide plans and suicide attempts was non-significant.

In this study, older age was statistically associated with suicidal ideation among all participants and associated with having a suicide plan among participants with suicidal ideation. In addition, being female and being single were statistically associated with suicidal ideation. In general, the suicide rate increases with age, and the highest rates among males and females are after 45 years and 55 years, respectively. ${ }^{24}$ Depression, physical illness, impaired functioning, and the death of a spouse have been known to contribute to suicide in old age. ${ }^{25}$ It has been suggested that the deterioration of cognitive and physical functions and the impairment of work capacity may affect the association between older age and suicidality among workers. Among females, suicidal ideation and suicide attempts tend to be three times higher than among males, although suicide completion is four times more prevalent among males. ${ }^{24}$ Among females as compared to males, more frequent suicidal ideation and behavior can be attributed to a twofold higher

Table 4. Results of hierarchical logistic regression analyses of participants with a suicide plan with a suicide attempt as the dependent variable $(\mathrm{N}=251)$

\begin{tabular}{|c|c|c|c|c|c|c|c|c|c|c|c|c|}
\hline \multirow{2}{*}{ Independent variables } & \multicolumn{3}{|c|}{ Model 1} & \multicolumn{3}{|c|}{ Model 2} & \multicolumn{3}{|c|}{ Model 3} & \multicolumn{3}{|c|}{ Model 4} \\
\hline & B & Wald & OR & B & Wald & OR & B & Wald & OR & B & Wald & OR \\
\hline \multicolumn{13}{|l|}{ Sociodemographic factors } \\
\hline Age (years) & -0.043 & 1.653 & 0.958 & -0.032 & 0.895 & 0.969 & -0.025 & 0.532 & 0.975 & -0.026 & 0.558 & 0.975 \\
\hline Gender (female) & 0.220 & 0.284 & 1.246 & 0.371 & 0.786 & 1.449 & 0.510 & 1.377 & 1.666 & 0.512 & 1.389 & 1.669 \\
\hline Marital status (single) & -0.133 & 0.104 & 0.875 & -0.220 & 0.277 & 0.802 & -0.262 & 0.390 & 0.769 & -0.258 & 0.374 & 0.773 \\
\hline Educational level (high) & -0.086 & 0.011 & 0.917 & -0.163 & 0.038 & 0.849 & -0.364 & 0.183 & 0.695 & -0.372 & 0.191 & 0.689 \\
\hline Job duration & 0.027 & 0.760 & 1.027 & 0.021 & 0.440 & 1.021 & 0.022 & 0.505 & 1.022 & 0.022 & 0.522 & 1.022 \\
\hline Job grade (high) & 0.276 & 0.293 & 1.318 & 0.339 & 0.426 & 1.404 & 0.447 & 0.684 & 1.563 & 0.452 & 0.695 & 1.571 \\
\hline \multicolumn{13}{|l|}{ Job stress } \\
\hline KOSS-SF & & & & 0.031 & 5.641 & $1.031^{*}$ & 0.024 & 2.766 & 1.024 & 0.025 & 2.787 & 1.025 \\
\hline \multicolumn{13}{|l|}{ Psychiatric symptoms } \\
\hline CES-D & & & & & & & -0.022 & 0.888 & 0.978 & -0.021 & 0.771 & 0.979 \\
\hline BAI & & & & & & & 0.054 & 5.915 & $1.056^{*}$ & 0.054 & 5.812 & $1.056^{*}$ \\
\hline \multicolumn{13}{|l|}{ Resilience } \\
\hline K-CD-RISC & & & & & & & & & & 0.002 & 0.033 & 1.002 \\
\hline \multicolumn{13}{|l|}{ Statistics of the model } \\
\hline$-2 \mathrm{LL}$ & \multicolumn{3}{|c|}{239.469} & \multicolumn{3}{|c|}{233.715} & \multicolumn{3}{|c|}{225.694} & \multicolumn{3}{|c|}{225.662} \\
\hline Model $\chi^{2}$ & \multicolumn{3}{|c|}{$2.601(\mathrm{df}=6)$} & \multicolumn{3}{|c|}{$8.356(\mathrm{df}=7)$} & \multicolumn{3}{|c|}{$16.376(\mathrm{df}=9)$} & \multicolumn{3}{|c|}{$16.409(\mathrm{df}=10)$} \\
\hline Step $\chi^{2}$ & \multicolumn{3}{|c|}{$2.601(\mathrm{df}=6)$} & \multicolumn{3}{|c|}{$5.754(\mathrm{df}=1)^{*}$} & \multicolumn{3}{|c|}{$8.020(\mathrm{df}=2)^{*}$} & \multicolumn{3}{|c|}{$0.033(\mathrm{df}=1)$} \\
\hline Nagelkerke $\mathrm{R}^{2}$ & \multicolumn{3}{|c|}{0.017} & \multicolumn{3}{|c|}{0.053} & \multicolumn{3}{|c|}{0.102} & \multicolumn{3}{|c|}{0.102} \\
\hline Classification accuracy & \multicolumn{3}{|c|}{81.3} & \multicolumn{3}{|c|}{81.3} & \multicolumn{3}{|c|}{81.7} & \multicolumn{3}{|c|}{81.3} \\
\hline
\end{tabular}

${ }^{*} \mathrm{p}<0.05,{ }^{* *} \mathrm{p}<0.01,{ }^{* * *} \mathrm{p}<0.001$. OR: odd ratio, KOSS-SF: Korean Occupational Stress Scale-Short Form, CES-D: Center for Epidemiologic Studies Depression scale, BAI: Beck Anxiety Inventory, K-CD-RISC: Korean version of the Connor-Davidson Resilience Scale 
prevalence of depression preceding suicide. ${ }^{26}$ Females also tend to express their distress through suicidal behavior without the intention to die and to report suicidal thoughts and plans more often than males. ${ }^{27}$ This may explain why suicide attempts are common in females but are less likely to lead to actual suicide as compared to males. Also, previous studies on gender differences in occupational stress have suggested higher occupational stress among females because of multiple and conflicting role demands at home and in the workplace. ${ }^{28,29} \mathrm{In}$ addition, female workers have been reported to experience high occupational stress because of their poorer job conditions compared to males, including low job grade, lack of decision latitude, long working hours, and high work strain. ${ }^{30,31}$ These gender differences in occupational stress may have been responsible for the increased suicidal ideation and plans in female workers in this study. In the general population, marriage significantly reduces the risk of suicide, while being single doubles it. ${ }^{24}$ According to Durkheim's social integration theory of suicide, marriage can reduce the risk of suicide by facilitating social support. In addition, by giving individuals an opportunity to care for another person, marriage encourages altruism and social integration. ${ }^{32}$ However, if this integration is broken through divorce and bereavement, the risk of suicide increases. ${ }^{33} \mathrm{~A}$ longitudinal study of large populations and a systematic review of 84 papers have supported this theory. ${ }^{33,34}$

In this study, a high level of job stress was statistically associated with suicidal ideation, but was non-significant with regard to suicide plans and attempts. A systematic review and meta-analysis ${ }^{6}$ examined the relationship between various work stressors and suicidal ideation, attempts, and completion. According to the results, a higher risk of suicidal ideation was associated with poor job support from supervisors and coworkers as well as work insecurity. Moreover, the higher risk of both suicide attempts and completion was associated with low job control. In addition, general self-reported occupational stress and exposure to work stressors were related to suicidal ideation, attempts, and completion. ${ }^{6}$

In this study, depressive mood and anxiety were statistically associated with suicidal ideation. In addition, a high level of anxiety was statistically associated with having a suicide plan among participants with suicidal ideation. It is known that about $95 \%$ of people who attempt or commit suicide have a diagnosed mental illness, and depressive disorder accounts for $80 \%$ of these people. ${ }^{24}$ In addition, the literature identifies severe anxiety and agitation as risk factors for suicide. Almost $20 \%$ of patients with an anxiety disorders such as panic disorder and social phobias make unsuccessful suicide attempts. ${ }^{24}$ Agitation was identified as one of the strongest predictors of short-term suicide in patients with depression and as one of the predictors of near-lethal attempts. ${ }^{35}$
In this study, resilience was a significant protective factor for suicidal ideation after adjusting for all other variables. Previous studies on substance-dependent patients have related low resilience to a high risk of suicide attempts. ${ }^{13}$ In a lifespan analysis study, low resilience was shown to predict a high likelihood of suicidal ideation for all ages in a community sample. ${ }^{14}$ Some researchers have proposed various definitions of suicide resilience- a capability, resource, or competitiveness to control suicidal ideation ${ }^{36}$ - and identified it as an internal factor protecting against suicidality. ${ }^{37}$ Further, one study contended that suicide resilience is a positive coping appraisal, which implies individual confidence in the ability to perform specific actions when dealing with difficult life situations. ${ }^{38}$ A subsequent study presented the buffering hypothesis of resilience against suicidality, in which resilience can moderate the impact of existing risk factors for suicidality, such as a negative attributional style, excessive perfectionism, and hopelessness, ${ }^{15}$ which may act as risk factors in the workplace as well. In this study, the association of resilience with suicide plans and attempts was nonsignificant. This result contradicted our hypothesis that high resilience among workers is related to a low incidence of suicide plans among participants with suicidal ideation and a low incidence of suicide attempts among participants with suicide plans. This non-significant influence beyond suicidal ideation can be understood based on suicidality as a continuum with suicidal ideation, plan, attempts, and completion. ${ }^{39}$

In addition to the major findings of this study, high job grade and high educational level were statistically associated with the absence of suicidal ideation in analysis models 1,2, and 3 of the hierarchical logistic regression analyses of all participants with suicidal ideation as the dependent variable, although they became statistically non-significant in analysis model 4 . Firstly, lower job grade may be associated with other adverse working conditions that increase suicidality, such as low control, monotony, high job strain, and workplace bullying. ${ }^{4-6}$ Regarding educational level, there is the implication that it is negatively associated with occupational stress among workers. ${ }^{40,41}$ A previous study with a large sample of workers in 16 European countries $(n=13,695)$ reported a consistent link between low educational levels and high work stress. ${ }^{29}$ Intellectual skills, which could be associated with educational level, play an important role in choosing adaptive coping strategies when faced with workplace stress. ${ }^{42}$ Intellectual strengths can provide psychosocial resources for individuals to experience an increased sense of control and self-efficacy and lower stress levels. ${ }^{43,44}$ Therefore, these play a role as protective factors for suicidality among workers. ${ }^{45}$

\section{Limitations}

Several limitations should be considered when interpreting 
the results of this study. First, we measured participants' suicidality using simple yes or no questions asking whether they had experienced suicidal ideation, had suicide plans, and made suicide attempts. There have been many instruments of varying character and length for assessing suicidal ideation or intent. ${ }^{46}$ Some include just a few elements and others contain more than 20 elements. As suicidal ideation and intent vary in severity and their degree is clinically meaningful, the dichotomous measurements used to assess suicidality in this study have limitations. In addition, because important independent variables, including KOSS-SF, CES-D, BAI, and K-CD-RISC scores, were used as continuous variables, we were unable to demonstrate their linear associations with suicidality, which could have been possible if suicidality had been measured by a rating instrument. However, there is controversy regarding the adequacy of the positive predictive value of suicidal behaviors using clinical instruments, even in more detailed questionnaires. ${ }^{47}$ In addition, non-suicidal self-injury could have been included as a group of people who attempt suicide in this study. Second, since the study used a self-reported survey, reporting bias may be a concern. Third, the current study did not collect data on various sociodemographic factors such as socioeconomic status, personal life stress outside work, and psychiatric comorbidities such as substance use disorder, impulse control disorder, and personality disorder, which may affect suicidality. Risk factors for suicidality including a family history of suicide and childhood abuse were also not examined in this study. Fourth, the current study did not collect data on various job-related factors such as work hours and workplace atmosphere, which can potentially influence suicidality. Finally, this study on resilience has the limitation of utilizing a crosssectional design. Resilience can be understood as having both "trait-like" and "state-like" characteristics. Therefore, causality here may be limited. ${ }^{8}$ Therefore, to confirm and generalize the results of this study, further longitudinal studies using a larger number of participants and objective assessment tools that control for latent confounding factors are needed.

\section{Conclusion}

In this study, we found that a high level of resilience was associated with a low incidence of suicidal ideation in Korean workers. Based on these results, we suggest the need to develop and implement interventions to improve resilience in the workplace, thereby protecting workers, especially those with a high level of work stress, from suicidal ideation. Future research should focus on the development of interventions to promote resilience among workers in the workplace.

\section{Acknowledgments}

We dedicate this paper to the spirit of the departed, Professor Se-Won Lim.

\section{Conflicts of Interest}

The authors have no potential conflicts of interest to disclose.

\section{Author Contributions}

Conceptualization: Sun Mi Kim, Hye Ri Kim. Data curation: Sang Won Jeon, Eun-Jin Kim. Formal analysis: Sun Mi Kim, Kyoung Joon Min. Funding acquisition: Young-Chul Shin. Investigation: Sang Won Jeon, Eun-Jin Kim. Methodology: Sun Mi Kim, Seo-Koo Yoo. Supervision: Young-Chul Shin, Kyoung Joon Min. Writing_original draft: Sun Mi Kim, Hye Ri Kim. Writing_review \& editing: Sang Won Jeon.

\section{ORCID iDs}

Sun Mi Kim

Hye Ri Kim

Kyoung Joon Min

Seo-Koo Yoo

Young-Chul Shin

Eun-Jin Kim

Sang Won Jeon

https://orcid.org/0000-0003-4131-0542 https://orcid.org/0000-0002-4147-4056 https://orcid.org/0000-0003-2866-0273 https://orcid.org/0000-0003-2023-5416 https://orcid.org/0000-0002-1884-4231 https://orcid.org/0000-0003-2134-6089 https://orcid.org/0000-0002-7828-3296

\section{REFERENCES}

1. KOSIS. Causes of death statistics. Available at: http://kosis.kr/statHt$\mathrm{ml} /$ statHtml.do? orgId=101\&tblId=DT_1B34E05\&vw_cd=MT Z T I T L E \& 1 is t_ id $=$ D $11 \&$ s e q N o $=\& 1$ a n g mode $=$ ko\&language $=$ kor\&obj_var_id $=\& i t m \_i d=\&$ conn_path $=M T$ ZTITLE. Accessed July 17, 2019.

2. Kinchin I, Doran CM. The economic cost of suicide and non-fatal suicide behavior in the Australian workforce and the potential impact of a workplace suicide prevention strategy. Int J Environ Res Public Health 2017;14. pii: E347.

3. Loerbroks A, Cho SI, Dollard MF, Zou J, Fischer JE, Jiang Y, et al. Associations between work stress and suicidal ideation: Individual-participant data from six cross-sectional studies. J Psychosom Res 2016; 90:62-69.

4. Schneider B, Grebner K, Schnabel A, Hampel H, Georgi K, Seidler A. Impact of employment status and work-related factors on risk of completed suicide. A case-control psychological autopsy study. Psychiatry Res 2011;190:265-270.

5. Leach LS, Poyser C, Butterworth P. Workplace bullying and the association with suicidal ideation/thoughts and behaviour: a systematic review. Occup Environ Med 2017;74:72-79.

6. Milner A, Spittal MJ, Pirkis J, Chastang JF, Niedhammer I, LaMontagne $\mathrm{AD}$. Low control and high demands at work as risk factors for suicide: An Australian national population-level case-control study. Psychosom Med 2017;79:358-364.

7. Ahern NR, Kiehl EM, Sole ML, Byers J. A review of instruments measuring resilience. Issues Compr Pediatr Nurs 2006;29:103-125.

8. Rutter M. Resilience as a dynamic concept. Dev Psychopathol 2012;24: 335-344.

9. Lee JK, Choi HG, Kim JY, Nam J, Kang HT, Koh SB, et al. Self-resilience as a protective factor against development of post-traumatic stress disorder symptoms in police officers. Ann Occup Environ Med 2016; 28:58.

10. Mealer M, Jones J, Newman J, McFann KK, Rothbaum B, Moss M. The presence of resilience is associated with a healthier psychological profile in intensive care unit (ICU) nurses: Results of a national survey. Int J Nurs Stud 2012;49:292-299.

11. Wild J, Smith KV, Thompson E, Bear F, Lommen MJ, Ehlers A. A prospective study of pre-trauma risk factors for post-traumatic stress disorder and depression. Psychol Med 2016;46:2571-2582.

12. Robertson IT, Cooper CL, Sarkar M, Curran T. Resilience training in the workplace from 2003 to 2014: A systematic review. J Occup Organ Psychol 2015;88:533-562. 
13. Roy A, Sarchiapone M, Carli V. Low resilience in suicide attempters. Arch Suicide Res 2007;11:265-269.

14. Liu DW, Fairweather-Schmidt AK, Roberts RM, Burns R, Anstey KJ. Does resilience predict suicidality? A lifespan analysis. Arch Suicide Res 2014;18:453-464.

15. Johnson J, Wood AM, Gooding P, Taylor PJ, Tarrier N. Resilience to suicidality: The buffering hypothesis. Clin Psychol Rev 2011;31:563-591.

16. United States Department of Labor. Standard Industrial Classification division structure. Available at: https://www.osha.gov/pls/imis/sic manual.html. Accessed July 24, 2019.

17. Lee E, Shin H, Yang Y, Cho J, Ahn K, Kim S. Development of the Stress Questionnaire for KNHANES: Report of Scientific Study Service. Cheongju: Korea Centers for Disease Control and Prevention; 2010.

18. Chang SJ, Koh SB, Kang D, Kim SA, Kang MG, Lee CG, et al. Developing an occupational stress scale for Korean employees. Korean J Occup Environ Med 2005;17:297-317.

19. Taber KS. The Use of Cronbach's alpha when developing and reporting research instruments in science education. Res Sci Edu 2018;48:12731296.

20. Radloff LS. The CES-D scale: A self-report depression scale for research in the general population. Appl Psychol Meas 1977;1:385-401.

21. Cho MJ, Kim KH. Diagnostic validity of the CES-D (Korean version) in the assessment of DSM-III-R major depression. J Korean Neuropsychiatr Assoc 1993;32:381-399.

22. Beck AT, Epstein N, Brown G, Steer RA. An inventory for measuring clinical anxiety: psychometric properties. J Consult Clin Psychol 1988; 56:893-897.

23. Baek HS, Lee KU, Joo EJ, Lee MY, Choi KS. Reliability and validity of the Korean version of the Connor-Davidson Resilience Scale. Psychiatry Investig 2010;7:109-115.

24. Sadock BJ, Sadock VA, Ruiz. P. Kaplan and Sadock's Synopsis of Psychiatry. Behavioral Sciences/Clinical Psychiatry (11th Ed). Philadelphia: Wolters Kluwer; 2015.

25. Lapierre S, Erlangsen A, Waern M, De Leo D, Oyama H, Scocco P, et al. A systematic review of elderly suicide prevention programs. Crisis 2011;32:88-98.

26. Freeman A, Mergl R, Kohls E, Szekely A, Gusmao R, Arensman E, et al. A cross-national study on gender differences in suicide intent. BMC Psychiatry 2017;17:234.

27. Hawton K. Sex and suicide. Gender differences in suicidal behaviour. Br J Psychiatry 2000;177:484-485.

28. Leineweber C, Baltzer M, Magnusson Hanson LL, Westerlund H. Work-family conflict and health in Swedish working women and men: A 2-year prospective analysis (the SLOSH study). Eur J Public Health 2013;23:710-716.

29. Koura U, Sekine M, Yamada M, Tatsuse T. Work, family, and personal characteristics explain occupational and gender differences in workfamily conflict among Japanese civil servants. Public Health 2017;153: 78-90.

30. Sjogren E, Kristenson M. Can gender differences in psychosocial factors be explained by socioeconomic status? Scand J Public Health
2006;34:59-68.

31. Sekine M, Tatsuse T, Kagamimori S, Chandola T, Cable N, Marmot M, et al. Sex inequalities in physical and mental functioning of British, Finnish, and Japanese civil servants: Role of job demand, control and work hours. Soc Sci Med 2011;73:595-603.

32. Durkheim E. Suicide: A Study in Sociology. Routledge; London 2005.

33. Kposowa AJ. Marital status and suicide in the National Longitudinal Mortality Study. J Epidemiol Community Health 2000;54:254-261.

34. Stack S. Suicide: A 15-year review of the sociological literature. Part II: Modernization and social integration perspectives. Suicide Life Threat Behav 2000;30:163-176.

35. Ribeiro JD, Bender TW, Buchman JM, Nock MK, Rudd MD, Bryan CJ, et al. An investigation of the interactive effects of the capability for suicide and acute agitation on suicidality in a military sample. Depress Anxiety 2015;32:25-31.

36. Osman A, Gutierrez PM, Muehlenkamp JJ, Dix-Richardson F, Barrios FX, Kopper BA. Suicide Resilience Inventory-25: Development and preliminary psychometric properties. Psychol Rep 2004;94:1349-1360.

37. Rutter PA, Freedenthal S, Osman A. Assessing protection from suicidal risk: Psychometric properties of the suicide resilience inventory. Death Stud 2008;32:142-153.

38. Johnson J, Gooding PA, Wood AM, Tarrier N. Resilience as positive coping appraisals: Testing the schematic appraisals model of suicide (SAMS). Behav Res Ther 2010;48:179-186.

39. Carter G, Reith DM, Whyte IM, McPherson M. Repeated self-poisoning: Increasing severity of self-harm as a predictor of subsequent suicide. Br J Psychiatry 2005;186:253-257.

40. Lunau T, Siegrist J, Dragano N, Wahrendorf M. The association between education and work stress: Does the policy context matter? PLoS One 2015;10:e0121573.

41. Michael G, Anastasios S, Helen K, Catherine K, Christine K. Gender differences in experiencing occupational stress: the role of age, education and marital status. Stress Health 2009;25:397-404.

42. Harzer C, Ruch W. The relationships of character strengths with coping, work-related stress, and job satisfaction. Front Psychol 2015;6:165.

43. Fredrickson BL. The role of positive emotions in positive psychology: The broaden-and-build theory of positive emotions. Am Psychol 2001; 56:218-226.

44. Hobfoll SE. Social and psychological resources and adaptation. Rev Gen Psychol 2002;6:307-324.

45. Kim HR, Kim SM, Hong JS, Han DH, Yoo SK, Min KJ, et al. Character strengths as protective factors against depression and suicidality among male and female employees. BMC Public Health 2018;18:1084.

46. Runeson B, Odeberg J, Pettersson A, Edbom T, Jildevik Adamsson I, Waern M. Instruments for the assessment of suicide risk: A systematic review evaluating the certainty of the evidence. PLoS One 2017;12: e0180292.

47. Carter G, Milner A, McGill K, Pirkis J, Kapur N, Spittal MJ. Predicting suicidal behaviours using clinical instruments: Systematic review and meta-analysis of positive predictive values for risk scales. Br J Psychiatry 2017;210:387-395. 